\title{
Food Process Industries: Island of Automation in Agriculture sector
}

\section{VV Agravat ${ }^{1 *}$, BC Khodifad ${ }^{2}$, C Matholiya $^{1}$, R Swarnkar $^{3}$ and N Kumar ${ }^{4}$}

${ }^{1}$ Research Scholar, Department of Farm Machinery and Power Engineering, Anand Agricultural University, India

${ }^{2}$ Research Scholar, Department of Processing and Food Engineering, Junagadh Agricultural University, India

${ }^{3}$ Professor and Head, Department of Farm Machinery and Power Engineering, Anand Agricultural University, India

${ }^{4}$ Associate Professor and Head, Dept. of Processing and Food Engineering, Anand Agricultural University, India

*Corresponding Author: VV Agravat, Research Scholar, Department of Farm Machinery and Power Engineering, Anand Agricultural University, India.

Received: November 18, 2019; Published: November 28, 2019

DOI: 10.31080/ASAG.2019.03.0729

Automation can be defined as the technology through which a process is executed with least man interference. In manufacturing plants, automation has been actively followed over 50 years and it will definitely continue to be so, even more aggressively, in future to serve us. The interest toward automation in food processing industries is mainly due to the tremendous development of computer hardware and software technologies. As computers occupy almost every aspect of our daily lives, the public at large has come to expect a high level of automation in every aspect of the manufacturing processes. Another reason for the industrial automation can be said that the shortage of labour in some type of work. For example, at the time of some process which produce extreme heat that cannot be bear by personnel the automation technology approaches there.

At present, automation in the food processing industries is becoming serious. Numerous factors are pushing it towards automation. Some of these factors are: (1) increase in competition worldwide, (2) demand of the buyer for higher quality foods, (3) need for maintaining hygiene and purity, (4) safety factors related to workers, and (5) easiness in developing different kinds of product. For example, the traditional visual human inspection of food quality assurance is uneven, inefficient and tedious. The computerised new technology ensures consistent and accurate food quality evaluation. Recently Cohan Sujay Carlos and Arpit Sharma, Banglore based Engineers developed Mechanical Chef which can prepare more than hundred recipes. It is a fully automatic robot and now it is also in the market as a product.
In case of automation technology in agricultural sector food process industries are quite progressive and can be described as "islands of automation". According to the Centres for Disease Control and Prevention, USA approximately $70 \%$ of all food borne disease is due to viruses spread by direct or indirect contact with infected persons. In case, poor hygiene, a worker can easily spread pathogens by touching the product.

However, quick advances in computer technology and increased expectations of consumers and regulatory agencies for enhanced food quality and safety have forced the food industry to implant automation of most manufacturing processes.

\section{Improved productivity}

Plant productivity may be defined as the quantity of end products manufactured per unit of operating parameters - plant size, number of workers, time of operation, etc. One plant reported a $30 \%$ increase in plant productivity using three discrete microprocessor-based controllers designed to perform all continuous loops connecting complex, integrated systems, valve meshing, and some sequencing.

Improved product quality

As previously described now consumers themselves are much aware about the quality of the food product hence it has become compulsion to maintain quality. Automation of processing maintains the food quality very well. 


\section{Improved profitability}

Increased profit is perhaps most important from the perspective of management.Improved product quality and productivity of the plant consequently makes profit to the owner of the plant. Hence, automation has become an integral part of the food process industries.

\section{Tools of automation}

Followings are the various tools of automation being used in food process industries:

\section{Computer vision systems}

A computer vision or machine vision system is used to measure some aspect of the results of the manufacturing process (e.g., shape, size, texture, location) that is analytic of the accuracy, efficiency, or quality of the process. Computer vision applications currently range from simple inspection to vision-guided robotic assembly. Most practical applications can be grouped into six general categories: Gauging, Verification, Flow detection, Identification, Recognition and Locating. For example, several commercial systems are being used to scrutinise 3.5 million pieces of fruit (apples, oranges, etc.) in an 8-h day. A researcher reported that on-line fillheight inspection system has been reported to handle speeds of up to 1400 bottles/min.

On-line sensors

\section{Electrically-based sensing}

A sensor, placed into an electrical circuit and it reacts with moisture, heat, pressure, or some other property in the food environment, or by chemical reaction with the food system, causing a disquiet in the electrical properties of the sensing material. This change can be measured and correlated with the attribute of interest.

\section{Physical sensing}

The physical sensing is done by measurement of physical quantities like temperature, pressure, weight, size and shape, fluid flow of gas and liquids and liquid level.

\section{Expert systems}

Neural network

A neural network is a system of hardware and/or software patterned after the operation of neurons in the human brain. Neural networks also called artificial neural networks are a variety of deep learning technology. ANN is capable of modeling complex nonlinear relationships and is highly climbable with parallel processing.
Applications of Neural Network in Food Processing

1. Control, Monitoring and Modeling of Industrials Processes

2. Recognition, Detection, Classification and Search for Patterns

3. Prediction On-Line Parameters

4. Image Processing

5. Optimization

6. Signal Processing

Fuzzy logic

Fuzzy logic is employed to handle the concept of partial truth, where the truth value may range between completely true and completely false. Fuzzy logic has been a valuable tool in dealing with unclear and incomplete information, and in incorporating human expert knowledge into process models. It has been executed in quality control for the food process industry.

Robot technology

Industrial robots are automated moving devices that can be deployed universally and that have multiple axes, the motion paths, sequences and angles of which can be freely programmed and controlled by sensors. In the early 1990's, the first applications in direct food handling in the bakery industry was approached. These robots were performing simple pick and place operations at a rate of 55-80 cycles per minute. The maximum use of robot technology is use for palletizing in food industry.

Checklist for automation in the food industries

A successful automation strategy rely on:

- $\quad$ Defining a future vision of the industry and its operations.

- Developing substitute automation scenarios.

- Defining criteria to evaluate each scenario

- Conducting a life-cycle cost study of competing the alternatives.

- Defining a coordinated implementation strategy.

Volume 3 Issue 12 December 2019

C) All rights are reserved by VV Agravat, et al. 